\title{
DYNAMICAL EVOLUTION OF THE ADEONA AND GEFION ASTEROID FAMILIES
}

\author{
V. CARRUBA and J. A. BURNS \\ Cornell University, Ithaca NY 14853, U.S.A. \\ W. BOTTKE and D. NESVORNÝ \\ Southwest Research Institute, Boulder CO 80302, U.S.A.
}

\begin{abstract}
In this work we investigate the degree to which large asteroid family fragments could be scattered by close encounters with the four most massive asteroids in the Main Belt (1 Ceres, 2 Pallas, 4 Vesta, and 10 Hygiea). We performed N-body simulations over $500 \mathrm{Myr}$ of members of the Adeona and Gefion families. While close encounters seem unable to significantly alter the semimajor axis distribution of these two families'members, single asteroids can be significantly moved from their original positions. These objects might be classified as interlopers if only based on their current values of apparent ejection velocity.
\end{abstract}

\section{Introduction}

Asteroid families are generally believed to result from the break-up of a parent body, with the smallest fragments traveling the furthest from the cluster's center (Cellino et al. 1999). One problem with this scenario is that the ejection velocities inferred for fragments from their observed dispersions in their current proper orbital elements seem to be consistently higher than those predicted by collisions in laboratory experiments and hydrocode simulations (Fujiwara et al. 1989, Benz and Asphaug 1999). In addition, larger fragments $(R>10 \mathrm{~km})$ show a typical "noise" or stochasticity in their ejection velocity fields of $30 \mathrm{~m} / \mathrm{s}$ ( $\Delta$ a of about $0.01 \mathrm{AU}$ in the central Main Belt, V. Zappalá 2001, private communication). These properties may be explained if family members were launched with low ejection velocities, and then diffused to their current locations. The Yarkovsky effect, i.e. the partial recoil that occurs as solar radiation absorbed on a small asteroid is asymmetrically re-emitted in the infrared band, could possibly be responsible for some asteroid mobility in proper element space. This effect is, however, basically negligible for asteroids with diameters of $50 \mathrm{~km}$ and greater, and more or less ineffective for bodies larger than $20 \mathrm{~km}$. Therefore, the Yarkovsky effect cannot provide the mobility needed to disperse the largest family members.

Here we investigate the degree to which large asteroid family fragments could be scattered by close encounters with the four most massive asteroids (1 Ceres, 2 Pallas, 4 Vesta, and 10 Hygiea). We performed N-body simulations over 500 Myr of members of the Adeona and Gefion families. These families were chosen

Celestial Mechanics and Dynamical Astronomy 00: 1-4, 2002.

(C) 2002 Kluwer Academic Publishers. Printed in the Netherlands.

article.tex; 11/02/2002; 13:54; p.1 


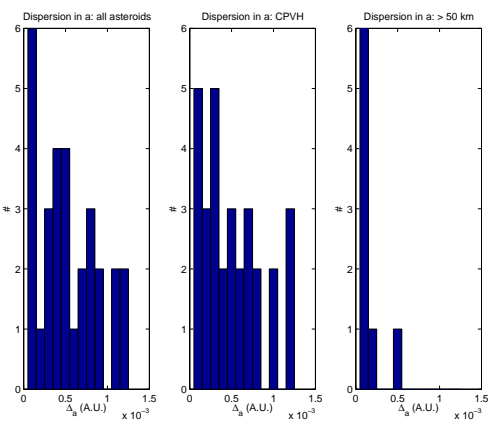

Figure 1. Histograms of changes in semimajor axis of 30 particles integrated over $100 \mathrm{Myr}$ under the influence of a) all 682 asteroids of radius larger than $50 \mathrm{Km}, \mathrm{b}) 1$ Ceres, 2 Pallas, 4 Vesta, and 10 Hygiea, and c) the remaining 678 bodies.

because they seem to result from a spherically symmetric ejection velocity field and they are close to 1 Ceres, the largest asteroid.

To learn how much of the semimajor axis mobility is due to close encounters with these four large asteroids, we ran a $100 \mathrm{Myr}$ integration of 30 test particles in the central Belt $\left(\mathrm{a}=2.6-2.8 \mathrm{AU}, \mathrm{e}=0.1, \mathrm{i}=0-15^{\circ}\right)$ under the action of $\left.\mathrm{i}\right)$ all 682 asteroids of radius larger than $50 \mathrm{~km}$, ii) 1 Ceres, 2 Pallas, 4 Vesta and 10 Hygiea, iii) the remaining 678 asteroids larger than $50 \mathrm{~km}$. To get our results, we used a Monte Carlo code capable of statistically treating $\Delta \mathrm{V}$ changes produced by close encounters between test bodies and large asteroids (Greenberg 1982). The gravitational interaction between two closely approaching bodies is modelled as a two-body hyperbolic encounter. While this approach is unable to account for secular effects and very close encounters (Dones et al. 1999), we believe that it is still reasonable to compare the relative importance of gravity assists from the four largest asteroids with those from the remaining 678 bodies larger than $50 \mathrm{~km}$. The changes in semimajor axis of the 30 particles are reported in figure 1 in the three cases. While the four largest asteroids moved 25 particles by more than $10^{-4} \mathrm{AU}$, with a maximum displacement of $1.2 \cdot 10^{-3} \mathrm{AU}$, the remaining 678 asteroids could move only 2 particles by more than $10^{-4}$ AU over the length of the integration. We therefore assumed that the four largest asteroids are the most effective perturbers in the central Belt, and neglected all the other bodies larger than $50 \mathrm{~km}$.

Next, we submitted the test particles to the gravitational effect of the four giant planets and the four most massive asteroids. Our integrations ran on the Cornell Supecomputer, and employed SWIFT-SKEEL (H. Levison personal communication), which symplectically integrates close encounters between a massless particle and a massive body. This algorithm combines a variant of the standard mixed-variable symplectic method (MVS, Wisdom and Holman 1991) with an improved version of the multiple time-step method originally developed by Skeel and Biesiadecki (1994). Further details can be found in Duncan et al. (1998). 


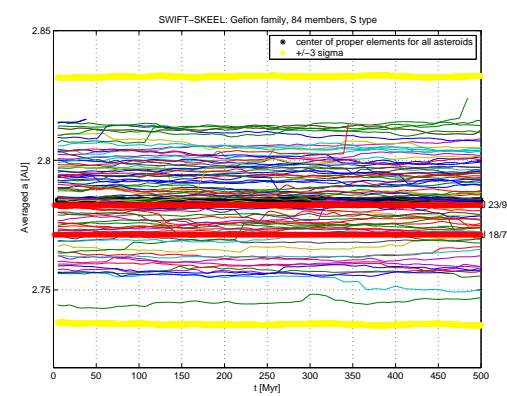

Figure 2. Time dependence of averaged $a$ of members of the Gefion family. Red lines indicate the position of mean motion resonances with Jupiter, the black line shows the center of the family and the yellow lines the 3 -sigma level in averaged $a$.

\section{Results}

For the Adeona and Gefion families of asteroids in the central Main Belt we wished to answer two questions about the long-term effects of close encounters:

- Can close encounters produce a significant dispersion in semimajor axis of family members, and be able to alter the dynamical shape of the family?

- What is the time behavior of changes in semimajor axis for members of these families? More precisely, if we express the standard deviation of changes in $a$ with time as:

$$
\sigma_{d a}=\alpha t^{\beta}
$$

with $\alpha$ a parameter depending on the initial dispersion, how does $\beta$ vary with time?

We integrated 84 members of the Gefion family and 66 members of Adeona. Members of the Gefion family not only sweep the same region of the Belt as 1 Ceres but also share the same inclination. This maximizes the probability of encounters, so we believe that the Gefion family should be the most affected by this phenomenon in the entire Belt. Our integrations ran for $500 \mathrm{Myr}$, and, in order to simply approximate the proper elements, we averaged the osculating elements with a running window of $10 \mathrm{Myr}$, and a shift of $10^{5} \mathrm{yr}$ (we call the result of this operation averaged elements).

Variation in the standard deviation of averaged $a$ at the end of the integration is less than $3 \%$ of the original value. This can be seen in figure 2, which reports the time dependence of averaged semimajor axis of Gefion members. The black line represents the center of the family and the yellow lines the three-sigma level. While several asteroids are displaced in $a$ by values up to $0.013 \mathrm{AU}$ at the end of the integration, the family shape is not significantly changed. If we plot diagrams of the $\log \mathrm{D}$ versus proper elements at the beginning and at the end (triangle diagrams), the global shape in $a$ is not significantly altered. Overall the triangles may represent the original velocity field, though it is also plausible that $\mathrm{D}<20 \mathrm{~km}$ bodies have 


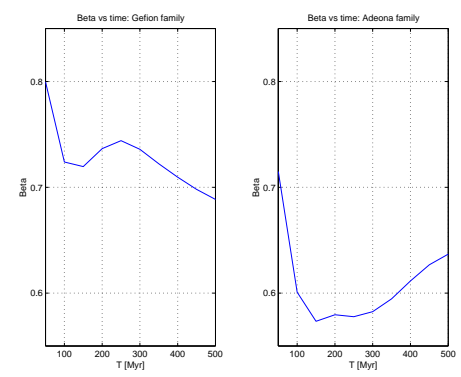

Figure 3. Time dependence of $\beta$ for the members of the Gefion and Adeona families.

had their initial orbits modified by collisions, close asteroid encounters, and the Yarkovsky effect.

About the second question, plots of $\beta$ versus $t$ for the Adeona and Gefion families are reported in figure 3. In agreement with Nesvorný et al 2001, that found a value of $\beta$ of 0.68 for asteroids in the central Belt, we find that changes in $a$ grow faster than a random walk law since there are encounters with repeated geometries, asymmetries in the perturbations, etc. Changes in semimajor axis are not described by a simple power law, they appear to have a more complicated relation with time. They also depend on the position of the asteroid family in the Main Belt (we found an average value for $\beta$ of 0.72 for Gefion and 0.61 for Adeona).

To conclude, close encounters with massive asteroids have not significantly changed the distribution in $a$ of the two simulated families. They can have however played a role in stimulating chaotic diffusion by injecting several members into mean-motion resonances, thereby changing the shape in $e$ and $i$ of the families, and can have significantly displaced a few asteroids from their original position, so changing their apparent ejection velocity.

\section{References}

Benz, W. and E. Asphaug 1999. Catastrophic disruption revisited. Icarus 142, 5-20.

Cellino, A., P. Michel, P. Tanga, V. Zappalá, P. Paolicchi, A. Dell'Oro 1999. The velocity-size relationship for members of asteroid families and implications for the physics of catastrophic collisions. Icarus 141, 79-95.

Dones, L., B. Gladman, H. J. Melosh, W. B. Tonks, H. F. Levison, and M. Duncan 1999. Dynamical lifetimes and final fates of small bodies: orbit integrations vs. Öpik calculations. Icarus 142, 509-524.

Duncan, M. J., H. F. Levison, and M. H. Lee 1998. A multiple time step symplectic algorithm for integrating close encounters. The Astron. J. 116, 2067-2077.

Fujiwara, A., P. Cerroni, D. R. Davis, E. Ryan, M. DiMartino, K. Holsapple, and K. Housen 1989. Experiments and scaling laws on catastrophic collisions. In Asteroid 2 (R. P. Binzel, T. Gehrels, and M. S. Matthews, Eds.), 240-265. Univ. of Arizona Press, Tucson.

Greenberg, R. 1982. Orbital interactions: a new geometrical formalism. Astron. J. 87, 184-195.

Nesvorný, D., A. Morbidelli, D. Vokrouhlický, W. Bottke, and M. Brož 2001. The Flora family: a case of the dynamically dispersed collisional swarm? Submitted to Icarus. 